\title{
The detection and treatment of human African trypanosomiasis
}

This article was published in the following Dove Press journal:

Research and Reports in Tropical Medicine

II June 2012

Number of times this article has been viewed

\author{
Bernard Bouteille' \\ Alain Buguet ${ }^{2}$ \\ 'Laboratory of Parasitology, Dupuytren \\ University Hospital of Limoges, France; \\ ${ }^{2}$ Polyclinic Marie-Louise Poto-Djembo, \\ Pointe-Noire, Congo
}

Abstract: Human African trypanosomiasis (HAT) is caused by the injection of Trypanosoma brucei (T. b.) gambiense or T. b. rhodesiense by Glossina, the tsetse fly. Three historical eras followed the exclusive clinical approach of the 19th century. At the turn of the century, the "initial research" era was initiated because of the dramatic spread of HAT throughout intertropical Africa, and scientists discovered the agent and its vector. Two entities, recurrent fever and sleeping sickness, were then considered a continuum between hemolymphatic stage 1 and meningoencephalitic stage 2. Treatments were developed. Soon after World War I, specific services and mobile teams were created, initiating the "epidemiological" era, during which populations were visited, screened, and treated. As a result, by 1960, annual new cases were rare. New mass screening and staging tools were then developed in a third, "modern" era, especially to counter a new epidemic wave. Currently, diagnosis still relies on microscopic detection of trypanosomes without (wet and thick blood films) or with concentration techniques (capillary tube centrifugation, miniature anion-exchange centrifugation technique). Staging is a vital step. Stage 1 patients are treated on site with pentamidine or suramin. However, stage 2 patients are treated in specialized facilities, using drugs that are highly toxic and/or that require complex administration procedures (melarsoprol, eflornithine, or nifurtimox-eflornithine combination therapy). Suramin and melarsoprol are the only medications active against Rhodesian HAT. Staging still relies on cerebrospinal fluid examination for trypanosome detection and white blood cell counts: stage 1 , absence of trypanosomes, white blood cell counts $\leq 5 / \mu \mathrm{L}$; stage 2 , presence of trypanosomes, white blood cell counts $\geq 20 / \mu \mathrm{L} ; T$. $b$. gambiense HAT intermediate stage, between these still controversial thresholds. Our group has proposed the use of noninvasive ambulatory polysomnography to identify sleep-wake abnormalities characteristic of stage 2 of the disease. Only patients with abnormal sleep-wake patterns would then undergo confirmative lumbar puncture.

Keywords: human African trypanosomiasis, sleeping sickness, Trypanosoma brucei gambiense, Trypanosoma brucei rhodesiense, diagnosis, lumbar puncture, polysomnography, treatment

\section{Introduction}

Human African trypanosomiasis (HAT), or sleeping sickness, is an endemic disease affecting 36 sub-Saharan countries. Sleeping sickness is a vector-borne parasitic disease with secondary invasion of the central nervous system (CNS), ${ }^{1,2}$ and it represents an excellent example of the "neglected disease" concept. ${ }^{3}$ The signs and symptoms of HAT remain unspecific and variable, which explains the difficulties in clinical diagnosis of the disease. This review, therefore, is focused on the clinical management, diagnosis, and treatment of HAT.
Correspondence: Bernard Bouteille Laboratory of Parasitology, Dupuytren University Hospital of Limoges, 2 avenue Martin Luther King, F-87042 Limoges Cedex, France

$\mathrm{Tel}+33555056160$

Fax+33555056177

Email bouteille@unilim.fr 


\section{Epidemiological background}

The causative agent of HAT is an extracellular trypanosome belonging to the Kinetoplastida order, Trypanosomatidae family, Salivaria section. The bloodstream form of African trypanosome (trypomastigote) is a parasite that dwells in body and tissue fluids - in particular, blood and cerebrospinal fluid (CSF). The spindle-shaped cell (12-42 by $1.5-3.5 \mu \mathrm{m})$ multiplies by longitudinal binary fission. Live trypanosomes are easily recognized because of their very active wiggling movements, due to the ondulatory motion of the flagellum, which runs along the cell body and ends in front in a free tip. In stained preparations, the locomotor apparatus, a central nucleus, and a subterminal particle - the kinetoplast - are easily observed (Figure 1).

Glossina sp., the tsetse fly, transmits trypanosomes by biting vertebrate hosts. The vector is found only in subSaharan Africa, between latitudes $14^{\circ} \mathrm{N}$ and $20^{\circ} \mathrm{S}$. Sixty million of the 400 million inhabitants are at risk for the disease. ${ }^{4}$ On the basis of mortality, HAT has the ninth rank among 25 infectious diseases in Africa. ${ }^{5}$ However, it ranks just behind malaria when disability-adjusted life years are considered (1.5 million). ${ }^{6}$

The generally chronic Gambian form of sleeping sickness, caused by Trypanosoma brucei (T. b.) gambiense subspecies, evolves fatally over a period of several months or years. Endemic to West Africa, the disease has recently reached epidemic status in Central Africa. Angola, Democratic Republic of Congo, Sudan, Chad, Congo, Central African Republic, and Uganda contribute to $98 \%$ of reported cases. ${ }^{7}$ The Glossina palpalis group is the main vector of

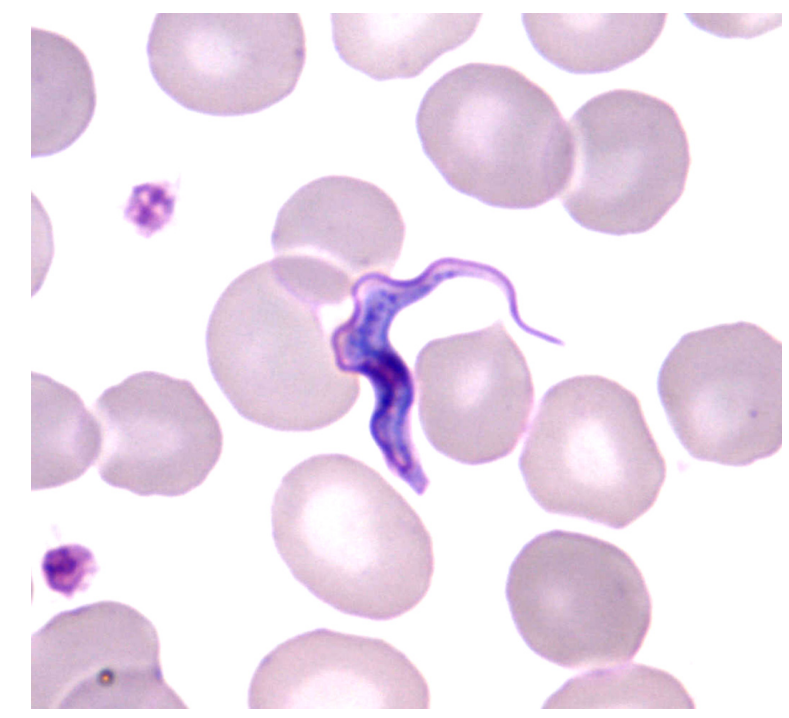

Figure I Trypanosoma brucei gambiense: bloodstream slender trypomastigote (thin smear, May Grunwald Giemsa, ×1000).
T. b. gambiense. Its habitat is represented by lake and river shores in forested or formerly forested areas; several species are also well adapted to areas of intensive agricultural activity. Man represents the reservoir of parasites, although both wild and domestic animals (mainly pigs and sheep) may be hosts for trypanosomes in some foci.

The acute Rhodesian form of the disease, caused by the morphologically identical $T$. b. rhodesiense, is found in East Africa (mainly Uganda and Tanzania). ${ }^{7}$ The Glossina morsitans group, or "game" tsetse fly, is its vector. It lives in the open woodland, bushland, or acacia thicket of the savannah zones. A wide spectrum of game animals and domestic cattle provides a reservoir of infection. Human infection is accidental and sporadic, depending on contact with the zoonotic cycle (hunters, firewood collectors, and tourists).

The natural history of HAT, following the 19th century clinical era, can be divided into three major historical periods in the domains of diagnosis and staging: between 1896 and 1911, especially in Uganda and the Congo basin, an "initial research" era; from the early 1920 s to the end of the colonial era, an "epidemiological" era; and from 1970 until present day, the "modern research" era. During the 19th century, HAT was rather sporadic, and medical doctors reported cases individually. By the turn of the century, however, the disease had become epidemic and killed an estimated one million people in the Congo basin and Uganda. ${ }^{8,9}$ British, Portuguese, Spanish, German, Belgian, and French colonial authorities commissioned medical research teams to Africa. In approximately ten years, the causal agent (the trypanosome) and its vector (Glossina) were identified, and diverse molecules were tested to treat patients (atoxyl, tryparsamide, salvarsan, etc). The control of the disease was then rapidly organized. Population screening developed immediately after World War I. Clinical descriptions lost their prominence. The elaboration of rapid biological tests and epidemiological mass screening techniques contributed to the quasi-disappearance of HAT at the end of the colonial era. However, due to political instability and loco-regional military unrest in Africa, a new epidemic wave hit the continent at the end of the 20th century. In 1998, the situation was catastrophic: 37,385 new cases were reported to the World Health Organization (WHO), and estimates varied from 300,000 to 500,000 cases. Unfortunately, less than $5 \%$ of the 60 million people at risk were under active surveillance. In several places, mortality was estimated at $50 \%$. Under leadership from the WHO, the international community, the pharmaceutical industry, foundations, and nongovernmental agencies mobilized against the epidemic. Recently, the "elimination" concept was revived, 
which led to the development of epidemiological surveillance systems and stressed the need for improving detection and treatment of new cases. These efforts were efficient, and the disease was again under control in $2006 .{ }^{10}$ In 2009 , for the first time in 50 years, the number of new cases reported to the WHO dropped below 10,000. ${ }^{11,12}$ Endemic countries in Central Africa reported 7,035 new cases in 2010. T. $b$. gambiense HAT represented $95 \%$ of the cases, but only $50 \%$ were detected by active prospection. ${ }^{13}$ Nevertheless, the real number of new cases per year may be much higher. ${ }^{14} \mathrm{~A}$ recent initiative has been the development of a geographic database for geolocation of the cases that have been detected either by active surveillance or passively. ${ }^{15}$ A HAT risk map, which demonstrates that historical foci are still active today, was established recently. ${ }^{16}$

\section{The diagnosis of HAT}

In countries endemic to $T$. $b$. gambiense, clinical symptoms are linked to host susceptibility and immune reaction. ${ }^{17}$

Major changes in the immune system have been described: lymphadenopathies, splenomegaly, and hypergammaglobulinemia. Immune mechanisms cannot eliminate all parasites causing immunopathological phenomena, such as autoimmune reactions and immunosuppression. Trypanosome-derived components (such as variant surface glycoprotein) and soluble factors are involved in the modulation of the immune system by acting on the synthesis of immune factors. In addition to their partial evasion from antibody-mediated destruction, African trypanosomes also have developed effective subversion strategies. They can actively disrupt normal antigenstimulated $\mathrm{T}$ cell activation and proliferation, and cytokine/ chemokine production by lymphocytes and macrophages. ${ }^{18}$

However, the mechanisms by which trypanosomes damage the CNS remain obscure. Laminin- $\alpha 4$ chains are permissive for lymphocytes and parasites crossing the bloodbrain barrier. This active passage seems to be facilitated by the great quantity of IFN- $\gamma$ produced through the action of the trypanosome-leukocyte triggering factor (trypanine) on CD8 ${ }^{+}$lymphocytes. ${ }^{19}$

\section{Clinical diagnosis}

The clinical presentations of both the Gambian and Rhodesian forms of HAT include many variable and nonspecific signs or symptoms. Conventionally, the two successive stages occur soon after the initial chancre that develops at the site of inoculation. ${ }^{20}$ The chancre is a hot, edematous, erythematous, and tender nodule. Its diameter varies from a few millimeters to several centimeters. More frequently observed in T. b. rhodesiense HAT, it disappears within two to three weeks. ${ }^{21}$

The time course of the disease depends on the infectious agent. The Gambian form is chronic, lasting several months to years, which gives time for the patient to develop the whole panel of neurological signs. In the absence of treatment, death occurs anywhere from several months to years. The Rhodesian form of the disease is acute and, if untreated, death may occur in several weeks to months. However, nonconventional evolutions of either form of the disease can be observed: asymptomatic to acute infections for T. b. gambiense HAT and, conversely, chronic forms of T. b. rhodesiense HAT. ${ }^{22-25}$

The disease is classically divided into two successive phases. ${ }^{26}$ The hemolymphatic stage 1 follows the initial inoculation chancre and represents the parasitic invasion phase of the lymphatic organs. It is followed by the intrusion of parasites into the CNS, resulting in stage 2 meningoencephalitis that evolves towards pre-terminal demyelization encephalitis. $^{27}$

\section{Stage I - hemolymphatic stage}

Within a few days of the tsetse bite, fever develops, due to the invasion of the blood by trypanosomes and the elaboration of immune reactions by the host. This stage is characterized by the response of the reticuloendothelial system. General signs appear quickly, with accompanying fever, adenopathies, splenomegaly, hepatomegaly, skin rashes or trypanides, pruritus, and other signs of mobilization of the immune system (pro-inflammatory cytokines), affecting the heart and CNS. Irregular febrile episodes are accompanied by headaches, malaise, fatigue, anorexia, extreme thirst, muscle and joint pains, pruritus, anemia, rash, and often, deep hyperesthesia (Kerandel sign; see below). The lymph nodes are generally enlarged (adenopathies), of rubbery consistency, mobile, and painful at the beginning. These general signs persist for months or even years, with latent periods. Palpation is part of the diagnostic procedure in T. b. gambiense-infected areas. It is used to search for enlarged lymph glands at the sus-clavicular and posterior cervical regions (Winterbottom sign). The presence of adenopathies is of considerable diagnostic interest in any feverish patient living in or coming from an endemic zone. Pruritus will extend and become more intense, with scratching-induced skin lesions. Edema of the face and extremities is quite common early in the disease course. Minor neurological and endocrine disorders may appear before any change is detectable in the CSF, indicating 
that trypanosomes have not yet invaded the CNS. They are related to the flood of pro-inflammatory cytokines and the invasion of the hypophysis area, respectively. The endocrine syndrome is marked by a permanent feeling of being cold and by a disturbance of vegetative and sexual functions, characterized by lack of appetite or, in contrast, hyperphagia, polydipsia, loss of libido, and impotence or amenorrhea. Psychiatric signs will often appear. The patient becomes irritable and his/her behavior and professional life suffer from mood disorders or personality changes. These signs are especially noticed by the patient's family, colleagues, and friends. Cardiac abnormalities, ranging from tachycardia to pericarditis or myocarditis, can occur in Gambian forms. However, they are more often observed in Rhodesian infection, causing early patient death. ${ }^{28}$

\section{Stage 2 - meningoencephalitic stage}

Depending on the causal trypanosome, the meningoencephalitic stage develops insidiously over several months or a few years (slower time course for T. b. gambiense than T. b. rhodesiense infection). Most clinical signs are reversible after treatment, attesting to the involvement of reversible inflammatory lesions that may become irreversible after the installation of demyelization. The general signs of the hemolymphatic stage do not completely disappear: peaks of fever persist, while adenopathies and splenomegaly recede. The early neurological symptoms correlate with the widespread meningeal inflammation that develops in both forms of HAT. The classic picture of mental disturbances is the astonishing disinterest and indifference to the environment. The patient is apathetic and sometimes agitated, with antisocial or aggressive behavior, sudden anger, and episodes of confusion or delirium. Patients with sleeping sickness have been found in psychiatric institutions, with their disease undiagnosed. ${ }^{29}$ However, selective CNS localizations may explain the principal clinical neurological signs:

- Hypothalamus, especially supraoptic nuclei and suprachiasmatic nuclei: sleep and wake alternation disorders. A state of apathy and indifference, evoking disorders of the awakening processes, overcomes the patient, with drooping of the eyelids and sudden drops in neck muscle tone.

- Striatum involvement, explaining extrapyramidal signs.

- Thalamus and related structures: deep sensory disturbances, with hyperpathia, are notable complaints from patients. The peculiar deep hyperpathia (Kerandel sign) is almost pathognomonic; pain immediately following soft-tissue compression is out of all proportion to the exerted force.
- Primitive reflexes (palmomental reflex, sucking reflex) or Babinski sign also may be present. Disorders of tone and mobility, as well as abnormal gait and movements, are consistent features of the disease.

- Median eminence and hypophyseal-hypothalamic region: endocrine dysfunction, already reported at stage 1 . Loss of libido, impotence, amenorrhea, and infertility are usually reported. Sensations of hunger and thirst are disturbed, often excessive, in contrast to the poor general state of malnutrition of the patients.

The terminal encephalitis phase corresponds to the installation of demyelization and atrophy. These may be observed with encephalography and, more recently, by magnetic resonance imaging (MRI) and computed tomography. ${ }^{30}$ The abnormalities revealed by MRI can be found in other diseases as well, such as leukoencephalitis, progressive multifocal leukoencephalopathy, cerebral gliomatosis, acute demyelizating encephalomyelitis, adrenoleukodystrophy, metachromatic leukodystrophy, lymphoma, and tuberculosis. Because MRI is not readily available in endemic countries, it is not useful for the diagnosis of HAT. ${ }^{31}$ The patient will present an exacerbation of consciousness disturbances, dementia, with incoherence and incontinence, and epileptic crises. Finally, the general state deteriorates, and the patient succumbs, with progressive cachexia and physiological marasmus.

\section{Neurophysiological aspects}

Loss of muscle tone, especially rapid drops in neck muscle tone, is evocative of cataplexy, which may be related to the narcoleptic-like occurrence of rapid eye movement (REM) sleep during wakefulness. ${ }^{32}$ Excessive daytime sleepiness and nocturnal insomnia characterize the HAT patient; there is a disappearance of the circadian rhythm of the sleep-wake cycle. REM/nonREM sleep cycles become shorter and more spread out over a 24-hour period. Eventually, sleep episodes last only 80-90 minutes, and they are interrupted by wake episodes also lasting 80-90 minutes. There is no difference between night and day. Sleep episodes are often initiated by REM sleep followed by slow-wave sleep, instead of the reverse sequence, which is observed in healthy people. Briefly, the polysomnographic (PSG) syndrome described by our group ${ }^{32}$ consists of two symptoms: circadian deregulation of the sleep-wake alternation and sleep episode disruption, with sleep onset REM periods (ie, sleep episodes with a short REM latency).

Other biological rhythms are disturbed in patients with HAT: body temperature, with either hyper- or hypothermia; cortisol, prolactin and growth hormone release, and renin 
activity - these hormones lose their circadian rhythmicity. ${ }^{33-35}$ However, melatonin, the pineal hormone that interferes with the biological clock, remains undisturbed, except for a two-hour phase advance. ${ }^{36}$ The reversibility of sleep-wake disorders and hormonal secretions, as well as the phase advance of melatonin secretion, suggests the involvement of the pontine serotonergic nucleus raphe dorsalis in the deregulation of the internal clock. ${ }^{37}$

Noninvasive recording of the sleep-wake cycle using polysomnography, the conventional field-compatible technique to study sleep and wakefulness, was adopted as a potential means to determine CNS invasion. Indeed, our team demonstrated that the presence of a PSG syndrome, such as described above, is observed in stage 2 meningoencephalitic patients. Therefore, the idea that it might be performed in place of the lumbar puncture and CSF examination is supported ${ }^{38}$ Polysomnography, therefore, may be regarded as a noninvasive marker of stage 2 HAT. Only patients presenting a PSG syndrome at the staging diagnostic step or throughout the post-treatment followup would subsequently undergo confirmative lumbar puncture and CSF examination. The use of noninvasive polysomnography could significantly decrease the number of invasive diagnostic and followup lumbar punctures..$^{39}$ The latter are feared by the populations in the endemic areas; they try to avoid meeting with mobile teams during screening campaigns.

\section{Biological diagnosis}

Clinical examinations in the field are rare today; they have been replaced by biological diagnosis techniques, which are indispensable for the determination of which drug to use, because of the particular aspects presented by stage 2 drugs. Therefore, it is crucial to develop precise and specific diagnosis techniques-if possible, noninvasive-to distinguish between the two stages of the disease (staging). Passive screening of all patients consulting in a dispensary will not solve the problem: patient screening in endemic T. $b$. gambiense areas must be performed systematically and actively by mobile teams. The widespread use of screening techniques, such as card agglutination trypanosomiasis test (CATT), allows the identification of CATT-positive people, who are then suspected of being infected. In those suspects, blood and CSF are collected to search for parasites and identify infected individuals. However, mobile teams working in remote African villages use a panoply of 30-year-old biological tests, along with clinical examinations. Diagnosis is even more difficult, and often delayed, for patients presenting (passive detection) to healthcare facilities, especially in nonendemic areas. ${ }^{40-42}$ The diagnosis procedure consists of two successive steps screening and staging. Figure 2 shows an example of the diagnosis and staging tree used by our team in Congolese T. b. gambiense-infected areas.

\section{Screening}

The first step in screening a village population is to identify infected individuals carefully. Mass screening of the Gambian form uses the field-adapted CATT serological technique. The test is performed on whole blood sampled in capillary tubes from fingertip puncture, and it may be followed by plasma dilutions to improve specificity. ${ }^{43}$ Whole-blood CATT sensitivity is high (87\%-98\%). Therefore, negative results are highly indicative of the absence of trypanosome infection.

Public health strategies are being developed to establish CATT plasma dilution cut-offs to define "diseased cases," depending on disease prevalence. ${ }^{14}$ In Congo, a positive CATT finding at a 1:4 plasma dilution requires parasitological confirmation, despite a reported $95 \%$ specificity. In high prevalence areas $(>1 \%), 1: 16$ CATT plasma positivity may be regarded as indicative of infection, despite nondetection of blood or lymph node parasites. Such cutoffs are useful in mass screening, but they are not usable for individual hospitalized patients. There are other limits to the procedure. Cross-reaction with other parasites (microfilariae, plasmodia) may elicit false CATT positivity. Similarly, immunological screening does not constitute the absolute proof of active infection, as CATT is still positive three years after treatment. However, CATT remains the first intention technique used by mobile teams for HAT screening in T. b. gambiense-infected areas.

The diagnosis of certitude for HAT requires serologic positivity, followed by direct microscopic parasite detection in the blood and/or adenopathies. However, T. b. gambiense parasitemia is generally low and varies widely from day to day. As such, examinations should be repeated, which is often unrealistic in the field. Trypanosome fragility requires immediate examination to avoid false negative findings. Techniques for concentration of trypanosomes in blood samples consist of capillary tube centrifugation, ${ }^{44}$ the most used technique in field conditions; the expensive acridine orange quantitative buffy coat technique; ${ }^{45}$ and the miniature anion-exchange centrifugation technique, ${ }^{46-48}$ the most sensitive technique. Blood trypanosome detection is improved by red blood cell lysis, centrifugation, and pellet light-emitting diode fluorescence microscopy. ${ }^{49}$ 


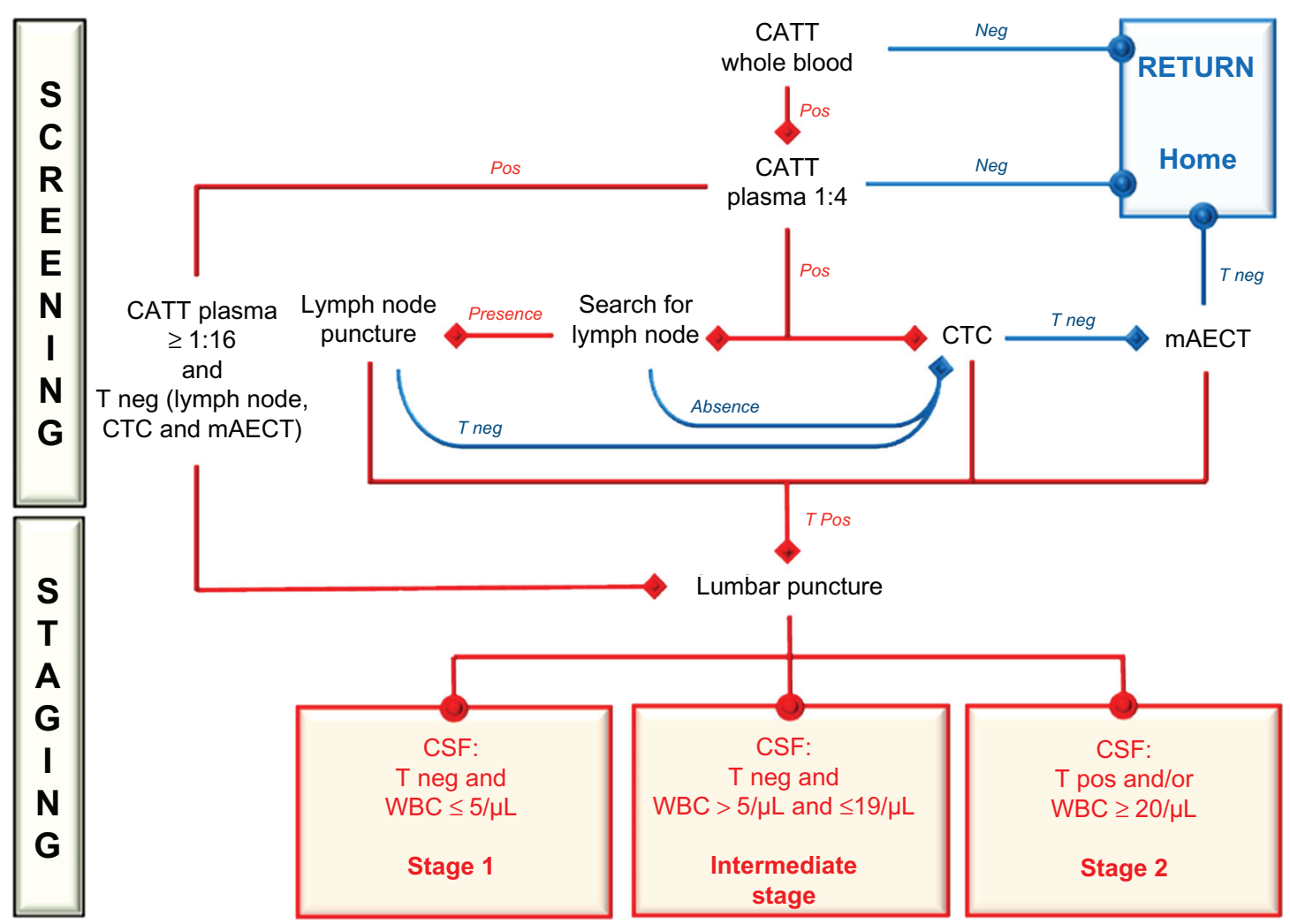

Figure 2 Decision tree for the conventional biological diagnosis and staging of $T$. b. gambiense human African trypanosomiasis, used by our team in the Congo, from 2005 to $2009 .^{39}$

Notes: Villagers were submitted to whole blood card agglutination trypanosomiasis test (CATT). CATT positive samples (suspects) were tested for CATT on plasma at a 1:4 dilution. CATT plasma I:4 negative people were released. CATT positive suspects were examined for the presence of trypanosomes to identify infected individuals. If present, the lymph nodes were punctured, and the fluid was examined microscopically. If trypanosomes were found, the suspect was classified as being a patient. In the absence of lymph nodes, blood trypanosome concentration techniques were undertaken. Capillary blood was centrifuged; if the capillary centrifugation technique (CTC) result was negative, a new blood sample was withdrawn from a forearm vein and passed through the miniature anion-exchange centrifugation technique. If the latter test was also negative, the suspect was released. All parasitologically positive suspects were now regarded as patients. All patients underwent staging procedures consisting of a lumbar puncture, as well as cerebrospinal fluid (CSF) sampling and examination. For the National Sleeping Sickness Control Program of the Congo, CATT plasma $\geq 1: I 6$ dilution positive people are considered as patients, and they undergo lumbar puncture, even if they are negative for trypanosomes in the lymph nodes and blood. If trypanosomes are observed in the sample, the patient is identified as stage 2. A white blood cell (WBC) count is also routinely performed. Three staging possibilities are determined: $\leq 5 \mathrm{WBC} / \mu \mathrm{L}$ of CSF, stage I; $\geq 20 \mathrm{WBC} / \mu \mathrm{L}$ of CSF, stage 2 ; between 6 and $19 \mathrm{WBC} / \mu \mathrm{L}$, intermediate stage. The patient is then treated accordingly.

Abbreviations: T. b., Trypanosoma brucei; CATT, card agglutination trypanosomiasis test; Neg, negative; Pos, positive; T neg, trypanosome negative; T pos, trypanosome positive; CTC, capillary tube centrifugation; mAECT, miniature anion-exchange centrifugation technique; CSF, cerebrospinal fluid; WBC, white blood cell.

Trypanosoma $b$. rhodesiense infection requires direct microscopy for parasite detection, as CATT is not adaptable to Rhodesian HAT. ${ }^{43}$ However, parasitemia is often elevated, and concentration techniques may not be necessary to confirm a diagnosis.

Other techniques have been used for trypanosome detection. Immunofluorescence or ELISA tests are efficient for $T$. b. gambiense or T. b. rhodesiense antibody detection, but they are not currently used in endemic countries. DNA amplification techniques, examined for potential use as a diagnostic tool, are not easily applicable for routine field diagnosis. However, a field-adapted, loop-mediated isothermal amplification PCR may be relevant, especially for $T$. b. rhodesiense detection. ${ }^{50-53}$ In patients presenting themselves to health care facilities in nonendemic areas, diagnosis is even more difficult and often delayed, because of the unusual occurrence of the disease in such areas.

\section{Staging}

Once a HAT infection has been confirmed, it is crucial to determine the stage of the disease (staging) in order to prescribe proper treatment and limit the risk of nosocomial complications. Clinical examination cannot be solely applied for stage determination, as neurological symptoms are often silent. This is especially true during active field population screening, where few cases present clinically evocative signs. Staging still relies on a lumbar puncture (Figure 3), which is very unpopular among populations. The invasion of the CNS 


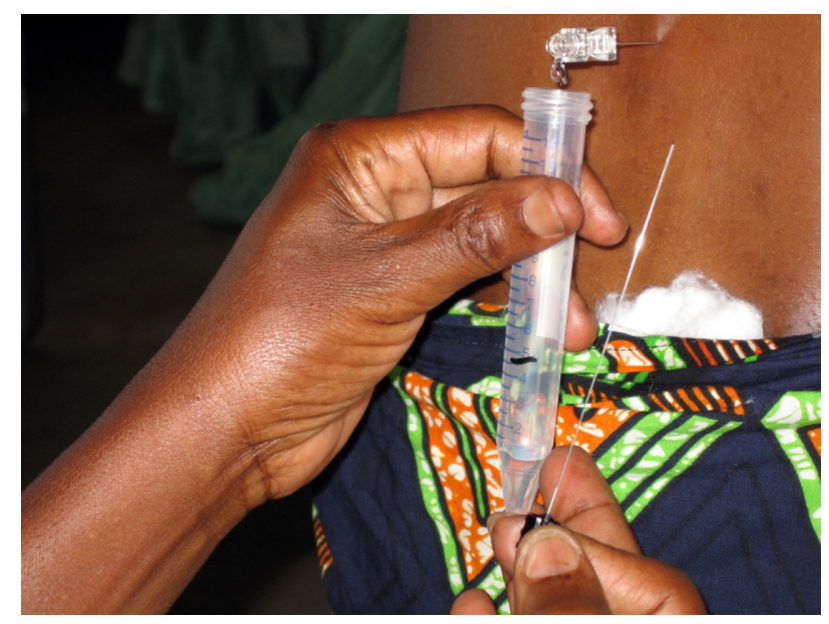

Figure 3 Lumbar puncture and cerebrospinal fluid (CSF) sample collection in a village healthcare facility. The patient gave her informed consent to be photographed and for the eventual publication of the picture.

is confirmed by the detection of trypanosomes in the CSF and/or increased white blood cell (WBC) counts, performed in diverse counting chambers (Nageotte, Neubauer, Malassez, $\mathrm{KOVA}^{\circledR}$, Uricult ${ }^{\circledR}$, etc).

For T. b. gambiense HAT, arbitrary cut-offs in WBC counts have been proposed in the absence of detectable trypanosomes in the CSF: stage 2 is diagnosed for $\geq 20 \mathrm{WBC} / \mu \mathrm{L}$; stage 1 is affirmed at $\leq 5 \mathrm{WBC} / \mu \mathrm{L}$; and, between these thresholds, an intermediate stage is widely accepted. However, the latter is seldom considered, because a carefully conducted followup may be difficult in endemic countries. It is often difficult to send the mobile team back to the place of the diagnosis, due to a lack of financial or logistic potential. In addition, patients may have moved away or refuse to attend the meeting. Therefore, cutoffs vary from one National Sleeping Sickness Control Program to another.

In the case of $T$. . . rhodesiense, the cutoff between stage 1 and stage 2 remains clearly at $5 \mathrm{WBC} / \mu \mathrm{L} \mathrm{CSF}{ }^{4}$ Unlike T. b. gambiense HAT, the staging of $T$. $b$. rhodesiense illness has not been studied recently. ${ }^{14}$

However, the WBC count may be versatile. A thorough search for trypanosomes requires a large enough CSF sample (at least 5-6 mL in adults and $2 \mathrm{~mL}$ in children); trypanosomes are rarely observed during cell counts, and simple or double centrifugations must be used to concentrate parasites. ${ }^{48,54}$

By examining WBC quality rather than quantity, our team developed a more precise staging technique, based on the identification of B lymphocytes in the CSF of T. b. gambiense HAT patients. ${ }^{55}$ Apart from cellular analyses, the CSF may be used to measure other unspecific and specific variables. For example, stage 2 patients exhibit elevated total protein concentration (more than $0.37 \mathrm{~g} / \mathrm{L}$ ) and increased immunoglobulin M levels, the magnitude of which is rarely observed, except in tuberculous meningitis, neurosyphilis, or subacute sclerosing panencephalitis. As such, a search for new biomarkers is ongoing. ${ }^{56-58}$

Recently, our team proposed the use of polysomnography as a noninvasive staging alternative. ${ }^{38}$ As stated above, stage 2 patients exhibit a PSG syndrome, with sleep-wake circadian disruptions and sleep-onset rapid eye movement sleep periods. During a TDR/WHO-funded five-year field investigation that was conducted in T. b. gambiense HAT foci in Congo Brazzaville, scalp and face electrodes were attached to 76 patients for 24-hour ambulatory recordings. ${ }^{39}$ Before treatment, stage 1 patients showed normal sleep-wake patterns. Stage 2 and several intermediate stage patients exhibited PSG disturbances. During post-treatment followup, the occurrence of PSG syndrome indicated relapse at stage 2. Our team concluded that noninvasive polysomnography might replace lumbar puncture for HAT staging, especially during post-treatment followup. The noninvasive polysomnography technique could be followed by CSF examination after lumbar puncture only in patients exhibiting a PSG syndrome.

\section{Treatment of HAT and post-treatment followup}

The few drugs currently used for the treatment of HAT (Table 1) are divided into two groups, based on their ability to cross the blood-brain barrier (melarsoprol, eflornithine, nifurtimox) or not (pentamidine, suramin). ${ }^{59}$ The mode of action of these drugs is still poorly understood, despite recent advances.$^{60}$ All anti-trypanosomal drugs are currently donated to WHO by Sanofi-Aventis Paris, France (pentamidine, melarsoprol, eflornithine) and Bayer Leverkusen, Germany (suramin, nifurtimox). Drugs are available from WHO (contact: simarrop@who.int).

\section{Treatment of stage I}

Treatment of stage 1 HAT is relatively simple and most often well tolerated. Intramuscular (IM) injections of pentamidine or suramin are currently administered under field conditions.

Pentamidine isethionate (Pentacarinat $\left.{ }^{\circledR}\right)$, an aromatic diamidine, is the drug of choice for stage $1 T$. b. gambiense HAT. Adverse effects have been more extensively studied in AIDS patients than in individuals with sleeping sickness. 
Table I Current therapies for stage I (hemolymphatic stage) and stage 2 (meningoencephalitic stage) of human African trypanosomiasis

\begin{tabular}{|c|c|c|}
\hline $\begin{array}{l}\text { Form of HAT } \\
\text { and stage }\end{array}$ & $\begin{array}{l}\text { First-line } \\
\text { treatments }\end{array}$ & \\
\hline \multicolumn{3}{|c|}{ Gambian form (T. b. gambiense) } \\
\hline Stage I & $\begin{array}{l}\text { Pentamidine } \\
\text { isethionate }\end{array}$ & $\begin{array}{l}4 \mathrm{mg} / \mathrm{kg} / \text { day IM (if IV: dilution in saline, } \\
2 \text {-h long infusion) } \times 7\end{array}$ \\
\hline Stage 2 & NECT & $\begin{array}{l}\text { Eflornithine } 2 \times 200 \mathrm{mg} / \mathrm{kg} / \text { day in } \\
\mathrm{I} \text {-hour long IV infusion } \times 7 \\
\text { plus nifurtimox } 3 \times 5 \mathrm{mg} / \mathrm{kg} / \text { day } \\
\mathrm{PO} \times 10\end{array}$ \\
\hline
\end{tabular}

\section{Second-line treatments \\ Treatments of therapeutic \\ failures and relapses}

Rhodesian form (T. b. rhodesiense)
$\begin{array}{ll}\text { Stage I } & \text { Suramin }\end{array}$

Stage 2

Melarsoprol
$4 \mathrm{mg} / \mathrm{kg} /$ day IM (if IV: dilution in saline,

Eflornithine $2 \times 200 \mathrm{mg} / \mathrm{kg} /$ day in

plus nifurtimox $3 \times 5 \mathrm{mg} / \mathrm{kg} /$ day

$P O \times 10$ a. Eflornithine $4 \times 100 \mathrm{mg} / \mathrm{kg} /$ day in I-hour long IV infusions $\times 14$

b. Melarsoprol $2.2 \mathrm{mg} / \mathrm{kg} /$ day IV $\times 10$

c. Three series of melarsoprol $3.6 \mathrm{mg} / \mathrm{kg} / \mathrm{day}$ IV $\times 3$ spaced by I-week intervals (not recommended)

Abbreviations: HAT, human African trypanosomiasis; T. b., Trypanosoma brucei; NECT, nifurtimox-eflornithine combination therapy; CSF, cerebrospinal fluid; WBC, white blood cells; IM, intramuscular; IV, intravenous; PO, per oral.

IM injections are painful. Nephrotoxicity, the most common complication, is generally reversible. The administration dosage is $4 \mathrm{mg}$ base per kg body weight. Courses of 7-10 IM injections (daily or on alternate days) are usually given; however, intravenous infusion (over 1-2 hours) is better tolerated. Patients should lie down for some time after each injection. Pentamidine treatment failures are rare; failures have been linked to staging errors. ${ }^{61}$

Suramin $\left(\right.$ Germanin $\left.^{\circledR}\right)$ is a sulphonated naphtylamine restricted to $T$. $b$. rhodesiense stage 1 infections. Adverse effects are numerous, including anaphylactic shock when there is concomitant filariasis. Nephrotoxicity is also commonly observed. However, discontinuation of the treatment will reverse any albuminuria or hematuria. Usually, 5-6 IM injections are administered at intervals of 5-7 days $(20 \mathrm{mg} / \mathrm{kg}$, with a maximum of $1 \mathrm{~g}$ per injection). Suramin resistance is also rare. Indeed, the metabolism of suramin does not seem to be a cause of resistance of $T$. $b$. rhodesiense, as suramin is extremely stable in vivo. ${ }^{62}$

\section{Treatment of stage 2}

The toxicity of molecules used to treat stage 2 and/or the difficulty encountered in their administration explain why patients at stage 2 are most often treated in hospital facilities. However, eflornithine treatment has been used successfully in some small bush hospitals.

Melarsoprol (Mel B, Arsobal ${ }^{\circledR}$ ) is a combination of the trivalent organic arsenical melarsen oxide with the heavy metal chelator British anti-Lewisite or dimercaprol. Melarsoprol is still the most powerful trypanocide available to cure both stages of $T$. $b$. gambiense and T. b. rhodesiense sleeping sickness. ${ }^{63}$ Currently, melarsoprol is not used to treat stage 1 infections. The highly irritating propylene glycol solvent imposes slow intravenous injections. Treatment regimen varies greatly: total doses of $1.26-2.16 \mathrm{~g}(35-60 \mathrm{~mL})$, intervals between series of 7-14 days, gradually increasing doses or maximum dose from the onset. The number of treatment series is either maximal as soon as staging indicates CNS invasion or increased in parallel with the CSF-leukocyte count. ${ }^{64}$ The most recent recommendation is to use a low dose of $2.2 \mathrm{mg} / \mathrm{kg}$ per day for 10 consecutive days. ${ }^{65}$ Complications may be severe, especially reactive arsenical encephalopathy. Generally accompanying the first or second series of injections, this syndrome begins with a rapid deterioration of consciousness, fever, and convulsions or status epilepticus, frequently leading to pulmonary edema, coma, and death. ${ }^{66}$ The continuous low-dose, 10-day treatment does not reduce the risk of reactive encephalopathy. Mortality affects $5 \%$ to $10 \%$ of patients. Neuropathological analysis reveals acute hemorrhagic leukoencephalopathy. ${ }^{67}$ Melarsoprol treatment cessation and administration of high doses of parenteral corticosteroids and anticonvulsive drugs should be undertaken as soon as CNS complications are suspected. Other adverse effects also may occur, such as peripheral polyneuropathy, headaches, fever, nausea, diarrhea, myalgia, cutaneous eruptions (including exfoliative dermatitis), 
Lyell syndrome (sometimes lethal), thrombophlebitis, cardiotoxicity, nephrotoxicity, hepatic dysfunction, and agranulocytosis. In addition, trypanosomes are becoming increasingly resistant to melarsoprol. Because of the risk of severe complications with melarsoprol administration, pentamidine treatment has been recommended in intermediate stage patients $(6-19 \mathrm{WBC} / \mu \mathrm{L}$ of $\mathrm{CSF})$, as for stage 1 patients $(\leq 5 \mathrm{WBC} / \mu \mathrm{L}$ of $\mathrm{CSF})$, despite pentamidine treatment failures in the former.

Alternative stage 2 treatment options are now available for T. b. gambiense HAT: eflornithine ${ }^{68,69}$ and, more recently, nifurtimox-eflornithine combination therapy (NECT). ${ }^{70}$ NECT has been recommended by WHO after numerous reports of melarsoprol resistance or treatment failures in foci with high prevalence for HAT, since the 1990s. ${ }^{12}$ Eflornithine is a specific, enzyme-activated, irreversible inhibitor of ornithine decarboxylase. The recommended schedule is $4 \times 100 \mathrm{mg} / \mathrm{kg} /$ day in 1 -hour IV infusions $\times 14$. However, eflornithine does not cure $T . b$. rhodesiense stage 2. Children experience a high relapse rate, presumably due to fast renal drug clearance. The following adverse effects have been reported: anemia, diarrhea, convulsions, vomiting, abdominal pain, headache, and alopecia. Convulsions generally occur during the first week of treatment.

Nifurtimox (Lampit ${ }^{\circledR}$ ) is a 5-nitrofuran used to treat American trypanosomiasis (Chagas disease) since the late 1960s. Reversible cerebellar syndrome (ataxia, nystagmus, tremors, and vertigo) may occur at doses of $30 \mathrm{mg} / \mathrm{kg} /$ day for 30 days. The WHO recommends NECT in T. b. gambiense HAT: IV eflornithine treatment is followed by oral administration of nifurtimox (eflornithine $2 \times 200 \mathrm{mg} / \mathrm{kg} /$ day in 1-hour $\mathrm{IV}$ infusion $\times 7$, plus nifurtimox $3 \times 5 \mathrm{mg} / \mathrm{kg} /$ day $\mathrm{PO} \times 10$ ). As the risk of adverse effects is lower with eflornithine and NECT than with melarsoprol, the former treatment procedures are recommended in patients with $6-19 \mathrm{WBC} / \mu \mathrm{L}$ of $\mathrm{CSF}$, due to pentamidine treatment failures in such intermediate stage patients. Resistance to nifurtimox and eflornithine is easily acquired in vitro. However, research is required to determine the emergence and spread of resistance and crossresistance in the field. ${ }^{71}$

\section{Post-treatment followup}

An 18-month to two-year post-treatment followup is necessary to declare a patient cured. Therefore, patients must have a lumbar puncture during each of the biannual examinations. A recent report stated that stage 2 followup could be shortened to a maximum duration of 12 months, based on CSF WBC counts. ${ }^{72}$ However, in our recent practice in Congo, several relapses were observed 18 months after proper treatment. ${ }^{39}$ Improvement in patient condition and resolution of the clinical and biological abnormalities indicate a good response to treatment. However, negative CATT findings are not indicative of a cure; therefore, CATT should not be used to monitor treatment outcome. ${ }^{73}$ Although PCR has been used along with parasite detection techniques for diagnosis and staging of T. b. gambiense HAT, it cannot be used during post-treatment followup, because of false positives after successful treatment, due to the persistence of DNA material from living or dead parasites. ${ }^{74}$ Relapse is marked by a new increase in the CSF WBC count, and patients should be retreated. When patients are treated early, complete recovery is the rule. In advanced cases, treatment stops the disease progression, but sequels are frequent.

\section{Conclusion}

Populations at risk for HAT live in remote bush villages and often experience poor financial conditions. Their isolation hampers adequate healthcare, and traditional practitioners often constitute first-line medicine. For many years, progress in HAT control has been very limited. ${ }^{75}$ Public health priorities need to ensure and enhance active detection of patients by well-equipped, motivated, mobile teams. Patient transportation to treatment centers, feeding, and nursing care also represent a major burden for financially demonetized villagers. Followup examinations should be conducted for at least two years to ensure treatment efficacy. Unfortunately, however, as the prevalence of the disease decreases, public funding vanishes, and, as regular surveys become sparse, the disease re-emerges. Furthermore, HAT management needs new diagnostic tests, especially noninvasive techniques, to improve stage determination and new treatment strategies to replace the old drugs that are used today.

\section{Acknowledgements}

Part of the work received support from Technical Services Agreement \# T7/83/2 of the World Health Organization (2005-2006), from the "Action de recherche en réseau: Le syndrome du cycle veille-sommeil dans la trypanosomiase humaine africaine: méthode non invasive de diagnostic du stade de la maladie, de validation de tests biologiques et de suivi de traitement" of the Agence universitaire de la Francophonie (2005-2006), and from the UNICEF/UNDP/World Bank/ 
WHO Special Programme for Research and Training in Tropical Diseases (TDR) N A50468 "Polysomnography, electrochemistry, immunology and neuroanatomy to the diagnosis of Human African Trypanosomiasis" (2006-2009).

Professor Emeritus Manny Radomski, University of Toronto, Ontario, Canada, kindly agreed to edit the manuscript.

\section{Disclosure}

The authors report no conflicts of interest in this work.

\section{References}

1. Kennedy PG. The continuing problem of human African trypanosomiasis (sleeping sickness). Ann Neurol. 2008;64(2):116-126.

2. Buguet A, Louis F, Bouteille B. Diagnostic de la trypanosomose humaine africaine (maladie du sommeil). Sarrebruck: Editions Universitaires Européennes; 2011.

3. Jannin J, Simarro PP, Louis FJ. The concept of neglected disease. Med Trop. 2003;63(3):219-221.

4. WHO. Control and surveillance of African trypanosomiasis. Report of a WHO Expert Committee. Technical Report Series $n^{\circ} 881$. Geneva. 1998.

5. Fèvre EM, Wissmann BV, Welburn SC, Lutumba P. The burden of human African trypanosomiasis. PLoS Negl Trop Dis. 2008;2(12): e333.

6. Hotez PJ, Fenwick A, Savioli L, Molyneux DH. Rescuing the bottom billion through control of neglected tropical diseases. Lancet. 2009; 373(9674):1570-1575.

7. Welburn SC, Maudlin I, Simarro PP. Controlling sleeping sickness a review. Parasitology. 2009;136(14):1943-1949.

8. Cox FE. History of sleeping sickness (African trypanosomiasis). Infect Dis Clin North Am. 2004;18(2):231-245.

9. Seed JR. African trypanosomiasis research: 100 years of progress, but questions and problems still remain. Int J Parasitol. 2001;31(5-6): 434-442.

10. WHO. Human African trypanosomiasis (sleeping sickness): epidemiological update. Wkly Epidemiol Rec. 2006;81(8):71-80.

11. Brun R, Blum J, Chappuis F, Burri C. Human African trypanosomiasis. Lancet. 2010;375(9709):148-159.

12. Simarro PP, Diarra A, Ruiz Postigo JA, Franco JR, Jannin JG. The human African trypanosomiasis control and surveillance programme of the World Health Organization 2000-2009: the way forward. PLoS Negl Trop Dis. 2011;5(2):e1007.

13. Ebeja AK, Valverde MO. Situation épidémiologique de la THA en 2010. Plateforme THA. Bulletin d'information. 2011;9:3-4.

14. Chappuis F, Loutan L, Simarro P, Lejon V, Büscher P. Options for field diagnosis of human African trypanosomiasis. Clin Microbiol Rev. 2005;18(1):133-146.

15. Simarro PP, Cecchi G, Paone M, et al. The atlas of human African trypanosomiasis: a contribution to global mapping of neglected tropical diseases. Int J Health Geogr. 2010;(9):57.

16. Simarro PP, Cecchi G, Franco JR, et al. Risk for human African trypanosomiasis, Central Africa, 2000-2009. Emerg Infect Dis. 2011;17(12): 2322-2324.

17. Bucheton B, MacLeod A, Jamonneau V. Human host determinants influencing the outcome of Trypanosoma brucei gambiense infections. Parasite Immunol. 2011;33(8):438-447.

18. Vincendeau P, Bouteille B. Immunology and immunopathology of African trypanosomiasis. An Acad Bras Cienc. 2006;78(4):645-665.

19. Masocha W, Rottenberg ME, Kristensson K. Migration of African trypanosomes across the blood-brain barrier. Physiol Behav. 2007;92(1-2): $110-114$.

20. Kennedy PG. Human African trypanosomiasis - neurological aspects. J Neurol. 2006;253(4):411-416.
21. Malvy D, Djossou F, Weill FX, Chapuis P, Longy-Boursier M, Le Bras M. Guess what! Human West African trypanosomiasis with chancre presentation. Eur J Dermatol. 2000;10(7):561-562.

22. Garcia A, Courtin D, Solano P, Koffi M, Jamonneau V. Human African trypanosomiasis: connecting parasite and host genetics. Trends Parasitol. 2006;22(9):405-409.

23. Truc P, Formenty P, Diallo PB, Komoin-Oka C, Lauginie F. Confirmation of two distinct classes of zymodemes of Trypanosoma brucei infecting man and wild mammals in Côte d'Ivoire: suspected difference in pathogenicity. Ann Trop Med Parasitol. 1997;91(8): 951-956.

24. Smith DH, Bailey JW. Human African trypanosomiasis in south-eastern Uganda: clinical diversity and isoenzyme profiles. Ann Trop Med Parasitol. 1997;91(7):851-856.

25. MacLean L, Chisi JE, Odiit M, et al. Severity of human African trypanosomiasis in East Africa is associated with geographic location, parasite genotype, and host inflammatory cytokine response profile. Infect Immun. 2004;72(12):7040-7044.

26. Checchi F, Filipe JA, Haydon DT, Chandramohan D, Chappuis F. Estimates of the duration of the early and late stage of gambiense sleeping sickness. BMC Infect Dis. 2008;8:16.

27. Blum J, Schmid C, Burri C. Clinical aspects of 2541 patients with second stage human African trypanosomiasis. Acta Trop. 2006;97(1):55-64.

28. Poltera AA, Cox JN, Owor R. Pancarditis affecting the conducting system and all valves in human African trypanosomiasis. Br Heart J. 1976;38(8):827-837.

29. Bedat-Millet AL, Charpentier S, Monge-Strauss MF, Woimant F. Psychiatric presentation of human African trypanosomiasis: overview of diagnostic pitfalls, interest of difluoromethylornithine treatment and contribution of magnetic resonance imaging. Rev Neurol. 2000;156(5): 505-509.

30. Sabbah P, Brosset C, Imbert P, Bonardel G, Jeandel P, Briant JF. Human African trypanosomiasis: MRI. Neuroradiology. 1997;39(10): 708-710.

31. Kager PA, Schipper HG, Stam J, Majoie CB. Magnetic resonance imaging findings in human African trypanosomiasis: a four-year follow-up study in a patient and review of the literature. Am J Trop Med Hyg. 2009;80(6):947-952.

32. Buguet A, Bourdon L, Bouteille B, et al. The duality of sleeping sickness: focusing on sleep. Sleep Med Rev. 2001;5(2):139-153.

33. Brandenberger G, Buguet A, Spiegel K, et al. Disruption of endocrine rhythms in sleeping sickness with preserved relationship between hormonal pulsatility and the REM - NREM sleep cycles. J Biol Rhythms. 1996;11(3):258-267.

34. Radomski MW, Buguet A, Montmayeur A, et al. Twenty-four-hour plasma cortisol and prolactin in human African trypanosomiasis patients and healthy African controls. Am J Trop Med Hyg. 1995;52(3): 281-286.

35. Radomski MW, Buguet A, Doua F, Bogui P, Tapie P. Relationship of plasma growth hormone to slow-wave sleep in African sleeping sickness. Neuroendocrinology. 1996;63(4):393-396.

36. Claustrat B, Buguet A, Geoffriau M, et al. Plasma melatonin rhythm is maintained in human African trypanosomiasis. Neuroendocrinology. 1998;68(1):64-70.

37. Buguet A. Is sleeping sickness a circadian disorder? The serotonergic hypothesis. Chronobiol Int. 1999;16(4):477-489.

38. Buguet A, Bisser S, Josenando T, Chapotot F, Cespuglio R. Sleep structure: a new diagnostic tool for stage determination in sleeping sickness. Acta Trop. 2005;93(1):107-117.

39. Buguet A, Bouteille B, Mpandzou G, et al. La recherche sur la maladie du sommeil (trypanosomose humaine africaine) en République du Congo de 2004 à 2009. Brazzaville: Editions Les Manguiers; 2009.

40. Migchelsen SJ, Büscher P, Hoepelman AI, Schallig HD, Adams ER. Human African trypanosomiasis: a review of non-endemic cases in the past 20 years. Int J Infect Dis. 2011;15(8):e517-e524. 
41. Simarro PP, Franco JR, Cecchi G, et al. Human African trypanosomiasis in non-endemic countries (2000-2010). J Travel Med. 2012;19(1):44-53.

42. Cottle LE, Peters JR, Hall A, et al. Multiorgan dysfunction caused by travel-associated African trypanosomiasis. Emerg Infect Dis. 2012; 18(2):287-289.

43. Magnus E, Vervoort T, Van Meirvenne N. A card-agglutination test with stained trypanosomes (C.A.T.T.) for the serological diagnosis of T. b. gambiense trypanosomiasis. Ann Soc Belg Med Trop. 1978;58(3):169-176.

44. Woo PT. Evaluation of the haematocrit centrifuge and other techniques for the field diagnosis of human trypanosomiasis and filariasis. Acta Trop. 1971;28(3):298-303.

45. Bailey JW, Smith DH. The use of the acridine orange QBC technique in the diagnosis of African trypanosomiasis. Trans $R$ Soc Trop Med Hyg. 1992;86(6):630.

46. Lumsden WH, Kimber CD, Evans DA, Doig SJ. Trypanosoma brucei: Miniature anion-exchange centrifugation technique for detection of low parasitaemias: Adaptation for field use. Trans R Soc Trop Med Hyg. 1979;73(3):312-317.

47. Lutumba P, Robays J, Miaka C, et al. Validity, cost and feasibility of the mAECT and CTC confirmation tests after diagnosis of African sleeping sickness. Trop Med Int Health. 2006;11(4):470-478.

48. Büscher P, Mumba Ngoyi D, Kaboré J, et al. Improved models of mini anion exchange centrifugation technique (mAECT) and modified single centrifugation (MSC) for sleeping sickness diagnosis and staging. PLoS Negl Trop Dis. 2009;3(11):e471.

49. Biéler S, Matovu E, Mitashi P, et al. Improved detection of Trypanosoma brucei by lysis of red blood cells, concentration and LED fluorescence microscopy. Acta Trop. 2012;121(2):135-140.

50. Kuboki N, Inoue N, Sakurai T, et al. Loop-mediated isothermal amplification for detection of African trypanosomes. J Clin Microbiol. 2003;41(12):5517-5524

51. Njiru ZK, Mikosza AS, Armstrong T, Enyaru JC, Ndung'u JM, Thompson AR. Loop-mediated isothermal amplification (LAMP) method for rapid detection of Trypanosoma brucei rhodesiense. PLoS Negl Trop Dis. 2008;2(1):e147.

52. Wastling SL, Picozzi K, Kakembo AS, Welburn SC. LAMP for human African trypanosomiasis: a comparative study of detection formats. PLoS Negl Trop Dis. 2010;4(11):e865.

53. Grab DJ, Nikolskaia OV, Inoue $\mathrm{N}$, et al. Using detergent to enhance detection sensitivity of African trypanosomes in human CSF and blood by loop-mediated isothermal amplification (LAMP). PLoS Negl Trop Dis. 2011;5(8):e1249.

54. Miézan TW, Meda HA, Doua F, Djé NN, Lejon V, Büscher P. Single centrifugation of cerebrospinal fluid in a sealed Pasteur pipette for simple, rapid and sensitive detection of trypanosomes. Trans $R$ Soc Trop Med Hyg. 2000;94(3):293.

55. Bouteille B, Mpandzou G, Cespuglio R, et al. Cerebrospinal fluid B lymphocyte identification for diagnosis and follow-up in human African trypanosomiasis in the field. Trop Med Int Health. 2010;15(4):454-461.

56. Hainard A, Tiberti N, Robin X, et al. A combined CXCL10, CXCL8 and H-FABP panel for the staging of human African trypanosomiasis patients. PLoS Negl Trop Dis. 2009;3(6):e459.

57. Kennedy PG. Novel biomarkers for late-stage human African trypanosomiasis - the search goes on. Am J Trop Med Hyg. 2010; 82(6):981-982.

Research and Reports in Tropical Medicine

\section{Publish your work in this journal}

Research and Reports in Tropical Medicine is an international, peerreviewed, open access journal publishing original research, case reports, editorials, reviews and commentaries on all areas of tropical medicine, including: Diseases and medicine in tropical regions; Entomology; Epidemiology; Health economics issues; Infectious disease; Laboratory
58. Geiger A, Simo G, Grébaut P, Peltier JB, Cuny G, Holzmuller P. Transcriptomics and proteomics in human African trypanosomiasis: current status and perspectives. J Proteomics. 2011;74(9):1625-1643.

59. Bouteille B, Oukem O, Bisser S, Dumas M. Treatment perspectives for human African trypanosomiasis. Fund Clin Pharmacol. 2003;17(2): 171-181.

60. Alsford S, Eckert S, Baker N, et al. High-throughput decoding of antitrypanosomal drug efficacy and resistance. Nature. 2012;482(7384): 232-236.

61. Paine MF, Wang MZ, Generaux CN, et al. Diamidines for human African trypanosomiasis. Curr Opin Investig Drugs. 2010;11(8):876-883.

62. Collins JM, Klecker RW Jr, Yarchoan R, et al. Clinical pharmacokinetics of suramin in patients with HTLV-III/LAV infection. J Clin Pharmacol. 1986;26(1):22-26.

63. Friedheim EAH. Mel B in the treatment of human trypanosomiasis. Am J Trop Med Hyg. 1949;29(2):173-180.

64. Neujean G. Contribution à l'étude des liquides rachidiens et céphaliques dans la maladie du sommeil à Trypanosoma gambiense. Ann Soc Belg Med Trop. 1950;30(5):1125-1387.

65. Schmid C, Richer M, Bilenge CM, et al. Effectiveness of a 10-day melarsoprol schedule for the treatment of late-stage human African trypanosomiasis: confirmation from a multinational study (IMPAMEL II). J Infect Dis. 2005;191(11):1922-1931.

66. Blum J, Nkunku S, Burri C. Clinical description of encephalopathic syndromes and risk factors for their occurrence and outcome during melarsoprol treatment of human African trypanosomiasis. Trop Med Int Health. 2001;6(5):390-400.

67. Adams JH, Haller L, Boa FY, Doua F, Dago A, Konian K. Human African trypanosomiasis (T. b. gambiense): a study of 16 fatal cases of sleeping sickness with some observations on acute reactive arsenical encephalopathy. Neuropathol Appl Neurobiol. 1986;12(1):81-94.

68. Milord F, Pépin J, Loko L, Ethier L, Mpia B. Efficacy and toxicity of eflornithine for treatment of Trypanosoma brucei gambiense sleeping sickness. Lancet. 1992;340(8820):652-655

69. Chappuis F, Udayraj N, Stietenroth K, Meussen A, Bovier PA. Eflornithine is safer than melarsoprol for the treatment of second-stage Trypanosoma brucei gambiense human African trypanosomiasis. Clin Infect Dis. 2005;41(5):748-751.

70. Priotto G, Kasparian S, Mutombo W, et al. Nifurtimox-eflornithine combination therapy for second-stage African Trypanosoma brucei gambiense trypanosomiasis: a multicentre, randomised, phase III, non-inferiority trial. Lancet. 2009;374(9683):56-64.

71. Barrett MP, Vincent IM, Burchmore RJ, Kazibwe AJ, Matovu E. Drug resistance in human African trypanosomiasis. Future Microbiol. 2011;6(9):1037-1047.

72. Mumba Ngoyi D, Lejon V, Pyana P, et al. How to shorten patient follow-up after treatment for Trypanosoma brucei gambiense sleeping sickness. J Infect Dis. 2010;201(3):453-463.

73. Lejon V, Ngoyi DM, Boelaert M, Büscher P. A CATT negative result after treatment for human African trypanosomiasis is no indication for cure. PLoS Negl Trop Dis. 2010;4(1):e590.

74. Deborggraeve S, Lejon V, Ekangu RA, et al. Diagnostic accuracy of PCR in gambiense sleeping sickness diagnosis, staging and posttreatment follow-up: a 2-year longitudinal study. PLoS Negl Trop Dis. 2011;5(2):e972.

75. Molyneux D, Ndung'u J, Maudlin I. Controlling sleeping sickness “when will they ever learn?” PLoS Negl Trop Dis. 2010;4(5):e609.

\section{Dovepress}

science and new technology in tropical medicine; Parasitology; Public health medicine/health care policy in tropical regions; and Microbiology. The manuscript management system is completely online and includes a very quick and fair peer-review system. Visit http://www.dovepress. com/testimonials.php to read real quotes from published authors. 